\title{
BMJ Open Quality Improving physical healthcare provided to psychiatric inpatients at an acute mental health trust
}

\author{
Shahnaz Hassan, Swetha Byravan, Hussain Al-Zubaidi
}

To cite: Hassan S, Byravan S, Al-Zubaidi H. Improving physical healthcare provided to psychiatric inpatients at an acute mental health trust. BMJ Open Quality 2019;8:e000537. doi:10.1136/ bmjoq-2018-000537

- Additional material for this paper are available online. To view these files, please visit the journal online (http://dx.doi.org/ 10.1136/bmjoq-2018-000537).

Received 30 0ctober 2018 Revised 9 April 2019 Accepted 24 July 2019

\section{Check for updates}

(c) Author(s) (or their employer(s)) 2019. Re-use permitted under CC BY-NC. No commercial re-use. See rights and permissions. Published by BMJ.

Psychiatry, Coventry and Warwickshire Partnership NHS Trust, Coventry, UK

Correspondence to Dr Shahnaz Hassan; shahna29@me.com

\section{ABSTRACT}

Psychiatric patients are at high risk of developing physical health complications. This is due to various factors including medications prescribed, life style choices and diagnostic overshadowing. Admission to a psychiatric unit provides a prime opportunity to review a patient's physical healthcare. We noticed prior to the commencement of this project that this opportunity was not always being used in the inpatient unit, with one in four patients at baseline data collection having no physical health checks. This is despite clear guidance laid out in the trust policy 'Physical Examination of Service Users during Admission to Hospital'. We aimed to improve compliance with these checks to $100 \%$. A number of prior audits in this area had failed to sustain improvement. Therefore, we proposed a quality improvement approach involving a series of plan do study act cycles, in order to test and review processes prior to implementation. The first cycle involved simplification of the paper-based documentation used for physical health checks, which resulted in minimal improvement by $5 \%$. The second cycle involved combining this documentation with the history taking proforma resulting in an overall improvement in compliance to $90 \%$. We learnt that a move away from the more widely used audit towards a more holistic approach of quality improvement allowed an informed continuum of change to take place which likely led to sustained improvement. Post implementation data collected at 1 month revealed compliance remained at $90 \%$. Our initial 100\% target was perhaps unrealistic, as there are also longstanding underlying cultural issues around physical healthcare in psychiatric patients that are complex to address and beyond the scope of this project.

\section{PROBLEM}

Coventry and Warwickshire partnership trust provides 234 acute inpatient psychiatric beds servicing approximately 850, 000 patients across the county. Junior doctors staff all inpatient units and they are also rostered to be on site out of hours, thus providing 24 hour care. When patients are admitted to the unit they require a physical health check including an examination, venous thromboembolism (VTE) assessment and baseline blood tests. Documentation of this is completed on a paper-based prepopulated proforma (see online supplementary Appendix 1a). This should be attempted for all admissions to ensure the patient is medically stable and there is no comorbid suspected medical cause for the psychiatric presentation. If the checks cannot be completed, for reasons such as aggression or high levels of agitation, it should be clearly documented and reattempted within the next $24-48$ hours by the next doctor on shift.

This process was not always followed and we noticed prior to commencement of this project that numerous patients had incomplete or no physical health checks done. It was not always clear if an assessment had been attempted and if incomplete whether there was a plan for a reattempt. Therefore, it was clear that there were missed opportunities to identify physical health needs. Lack of baseline information on a patient's health also meant that difficulties arose in providing continuous care.

Previous audits within the trust whereby changes were implemented without evidence of efficacy have failed to sustain sufficient improvements in compliance with completion of physical health checks. In order to understand the reasons behind this a survey was designed and disseminated to junior doctors prior to implementing the project. Prior to this, junior doctors had very little involvement in the design of the process of which they are paramount. We felt that involving them would be an essential part of generating effective improvement.

The aim was to increase the compliance with physical health checks to $100 \%$ with the intention of providing better physical healthcare for psychiatric inpatients, thereby improving the overall quality of care provided by the service. There are also long-term health gains for patients through identification and management of medical conditions. The intervention period was not predetermined.

\section{BACKGROUND}

It is well known that psychiatric patients are at particular risk of developing physical health 
complications when compared with non-psychiatric patients. Often mental illness has a negative relationship with physical health and those with severe mental illnesses are the most significantly affected. This is reflected in a London based study that revealed a 12.9-year reduction in life expectancy for males and 11.8-year reduction for females with a serious mental illness compared with the general UK population. ${ }^{1}$

There are a variety of reasons for this with lifestyle factors playing a major role. People with schizophrenia are 2.8-3.5 times more likely to be obese and therefore more likely to suffer from diabetes, hypertension and dyslipidaemia. ${ }^{2}$ The prevalence of smoking has also been reported to be up to $88 \%$ in this group, which increases the incidence of respiratory diseases. ${ }^{3}$ They are also more likely to adopt a sedentary lifestyle and a poorer diet when compared with the general population, ${ }^{4}$ this all culminates in cardiovascular disease being the leading cause of death in those with a serious mental illness, with depression being an independent risk factor itself. ${ }^{5}$

The higher rates of cardiovascular disease can also be attributed to psychotropic medications. There is an established link between antipsychotics and metabolic side effects such as obesity and diabetes especially with the use of clozapine. ${ }^{67}$ Patients with schizophrenia treated with antipsychotics have higher rates of both individual features of metabolic syndrome and the syndrome itself. ${ }^{8}$

There is also a relationship between sudden death and psychiatric patients, which can be attributed to a number of reasons. First of all, the rates of suicide are higher in patients with mental health issues especially in young schizophrenic patients. There also tends to be increased substance misuse among these patients, which is closely related to their mental health but this in turn doubles the risk of sudden death in those with affective and schizophrenic disorders. ${ }^{9}$

Another contributing factor for developing adverse health complications is the increased rates of risk taking behaviours either due to the mental illness itself or as a result of social circumstances. Patients with schizophrenia are more likely than non-psychotic individuals to contract human immunodeficiency or hepatitis $\mathrm{C}$ virus as a result of increased risk behaviours such as substance use and highrisk sexual behaviour. Overall adherence to prescribed medications in schizophrenia is inadequate and often these patients are unaware that they are infected and in such cases this would pose a public health risk. ${ }^{10}$

Doctors play an integral role in identifying and managing the health complications seen in psychiatric patients but unfortunately it is often these patients that are also overlooked. A retrospective study conducted in the primary care setting found that patients with schizophrenia were $50 \%$ less likely to have routine checks such as blood pressure and cholesterol than the general population or asthmatics. ${ }^{3}$ A UK-based study based on 106 outpatient psychiatric patients followed over 18 months found that $33 \%$ of patients had metabolic syndrome but monitoring of metabolic status and initiating intervention was poor. Only a maximum of $7.8 \%$ received lipid lowering medication and even after informing primary and secondary care physicians of the metabolic parameters they had either remained unchanged or worsened during follow-up. ${ }^{11}$ Monitoring and managing physical health complications among this group should be initiated by healthcare professionals since there is a significant associated cardiovascular morbidity but it is exactly these high risk patients who are neglected. ${ }^{12}$

Admission to an inpatient psychiatric unit is a key opportunity to review a patient's physical health. For some, this may be the first time encountering health services or the first in a while. It enables a thorough medical review to take place as well as an opportunity to educate and promote a healthy lifestyle. In order to achieve this, it is imperative to undertake a good medical clerking, conduct a physical examination and complete routine blood tests at the earliest opportunity. This creates a foundation for good medical care. The concern that patients with serious mental illness 'die earlier than the rest of the population due to preventable disease' and the opportunity that an inpatient admission provides to look at addressing this was identified by a Central and North West London NHS Foundation Trust. They were able to demonstrate that a quality improvement approach to creating a new physical health assessment form resulted in favourable outcomes. ${ }^{13}$

Therefore, given the increased risk of physical health complications in mental health patients at a cost to their quality and quantity of life it is imperative that these needs are identified and managed appropriately. Furthermore, those that require an admission are likely to suffer from more severe mental illness and therefore at greater risk of neglected physical health needs and diagnostic overshadowing. Admission provides a prime opportunity for these needs to be addressed by doctors through completion of the physical health proforma and hence work to improve the quality and compliance of this process will result in improved physical healthcare of local patients.

\section{BASELINE MEASUREMENT}

The standards for physical checks required on admission by doctors are delineated in the trust policy 'Physical Examination of Service Users during Admission to Hospital', available on the intranet. The policy details a prepopulated paper-based proforma (see online supplementary Appendix 1a) which doctors are required to complete as fully as able on admitting a patient to the ward. The proforma prompts and guides the doctor through the physical assessment and copies are made available to doctors at the point of admission on the wards. A peer already familiar with the process delineates these requirements to new doctors joining the trust at induction.

The policy stipulates that a detailed physical assessment consisting of a cardiovascular, respiratory, abdominal and neurological examination should be done within 
24 hours of admission. Physical observations which can be done by nursing staff including blood pressure, heart rate, temperature, oxygen saturations, respiratory rate and Glasgow coma scale are expected to be done within 6 hours. At the earliest possible opportunity a body map to identify scars, bruises, tattoos and piercings should be completed by the doctor and one other member of staff. VTE assessments should also be done for every patient as well as baseline bloods on admission.

If for any reason the doctor is unable to undertake a full physical health screen on the patient, then the reasons for this should be documented. If this is due to the patient refusing to engage as opposed to a risk concern by the doctor, then an assessment of their mental capacity to refuse should also be documented. For such patients where it is not possible to complete the full assessment as a bare minimum, a general screen should be undertaken. This includes an observation of the patient's physical state, for example, their mobility, alertness, overall wellness and measurement of physical observations if possible. Also, the doctor should review any collateral healthcare notes, which are often available to obtain details of their past medical history and allergy status for documentation.

For any patient where the process is not complete there should be a documented plan for reattempts. Data was collected against these standards set out in the trust policy using a spreadsheet tool designed on Microsoft Excel.

Baseline electrocardiogram (ECG) was not a mandatory trust requirement but was considered to be an important part of the physical assessment and there was a culture across the trust that this was done. We collected data to inform how many patients had ECGs completed and whether this was done as routine with the view of implementing this as a requirement.

Baseline data were collected over 3 months from December 2016 to February 2017 immediately after a new intake of junior doctors. All inpatient notes in the largest two sites across the trust were reviewed $(n=156)$. A total of 17 cases were excluded as the old proforma had been used. For patients where assessment was not done or incomplete, we looked at the case notes for documented reasons as to why this was not done, capacity assessments if applicable and if there was a plan for further follow-up.

Of these, $75 \%$ had a full physical health screen, $79 \%$ had base line bloods, $74 \%$ had a general screen and $71 \%$ had a completed VTE assessment. Of the cases where there was no physical examination, $85 \%$ had documented reasons for this, though these were not always appropriate, for example, patient asleep or routine job to be done by the ward teams. For patients where physicals were incomplete, $60 \%(\mathrm{n}=21 / 35)$ were documented to be refusing assessment but only $19 \%$ of these had a capacity assessment done. ECG was completed in $65 \%$ of cases.

An issue highlighted was a lack of written documentation of further plans to reattempt assessment in patients where this was not done or incomplete, with only $26 \%$ of these cases having a written plan. Incomplete assessments and lack of consideration of patient capacity to refuse assessment raise concerns about ethical conduct and patient safety.

Simultaneously, while collecting baseline data, we disseminated an electronic survey to seek the opinions of junior doctors in regards to the admission process and the physical health assessment. There were 14 responses in total. The survey demonstrated that while $100 \%$ of junior doctors agreed that physical health checks are a necessary part of the admission process, $45 \%$ did not find the process straightforward to complete. Many of the doctors responded that the admission proforma was repetitive and time-consuming resulting in poor completion of the forms. They also felt there were often barriers to completion, such as no transport system for blood tests done out of hours and lack of equipment, such as a working ECG machines.

\section{DESIGN}

The Model for Improvement developed by Associates in Process Improvement was used to design this project. The aim of the plan do study act (PDSA) cycles was to increase the compliance rate of physical health checks, to improve clinician awareness of the importance of these checks and the necessity to complete them at the point of admission or as soon as possible. The frequency of PDSA cycles was not predetermined and we were prepared to repeat cycles up until a point of satisfactory compliance was achieved, ideally to $100 \%$.

The quality improvement team consisted of one core psychiatry trainee (project lead) and one foundation trainee, the medical and nursing leads for safety and quality and the business change manager. Two other doctors, one foundation trainee and a one higher psychiatry trainee, were involved at periods in data collection, project design and presentation of the project at academic meetings.

\section{PATIENT AND PUBLIC INVOLVEMENT}

This project reviewed the doctor's compliance with the internal admission process so involvement of non-NHS staff was not required. Therefore, ethical approval was not required for the project to be approved by the safety and quality department.

\section{STRATEGY}

\section{First PDSA cycle}

At the initial stage of the first PDSA cycle, a multidisciplinary (MDT) meeting was held with safety and quality and clinical leads to discuss the baseline data gathered and review a proposed altered proforma that reflected the feedback of doctors. The content of the proposed proforma was largely the same but laid out in a more concise and intuitive way, removing repetition within the document. We also sought to make it clearer as to what is required when the assessment cannot be completed and included a flowchart for this. At the time of this project, 
the trust was using paper-based proformas though inpatient notes were electronic. The move to an electronic admission processes was in the pipeline and we collaborated with the business change manager for the trust to ensure that the proforma designed could be mapped into an online version.

This project and proposals were then presented at the weekly academic meeting and further feedback was obtained from colleagues. The new refined paper proforma (see online supplementary Appendix 1b) was subsequently piloted on two of the acute wards, one male and one female, and doctors were made aware of this via email, notices in the common rooms and on the respective wards. At the end of the proforma, we attached a paper-based questionnaire to gather feedback from junior doctors about their experience on filling out the new proforma and suggestions for further improvement.

The pilot took place over a 4-month period during which there was a changeover of junior doctors at 3 months into the pilot. At 4 months, the data were analysed and an overall improvement in compliance with the full physical health screen to $80 \%$ was noted but at an only $5 \%$ increase from baseline, this was marginal. There was, however, significant improvement noted in other aspects such as documentation of reasons why the proforma was incomplete and capacity assessment in patients that were not assessed, which went up from $19 \%$ to $80 \%$. We believe this was due to clear prompts being laid out on the first page contrary to the previous proforma where there was no clear direction on capacity assessments.

Feedback forms highlighted that doctors were largely finding the new proforma easier to use. However, it was clear that there still appeared difficulties in completing physical healthcare checks as measured by compliance. From peer group discussion, we learnt that junior doctors felt that some checks such as measurement of waist circumference, height, weight and body mass index (BMI) calculation were not required on admission but elongated the process. They felt the parent team or nursing staff could do this at a later point in the admission. Also, there were still practical issues with the transportation of blood test samples to the main hospital for processing and ECG machines not working. Furthermore, it was felt that there was still repetition within the process when combined with the history taking proforma as both ask for smoking status, drug, alcohol and medical history.

\section{Second PDSA cycle}

Subsequently, a further MDT meeting took place to plan the second PDSA cycle, which was to involve combination of the history taking and physical health proforma into a single paper document, universally formatted in the layout of the traditional medical review, further improving the intuitiveness of the process. Furthermore, by combining the assessments, there was a move towards more of a holistic approach. Given the perception by some medical staff that physical health on a mental health unit is not an urgent matter, a clear note was put on the front of the proforma detailing the expectation to attempt to complete the entire proforma including the physical health checks. Dispute between aspects that doctors felt could be removed such as calculation of BMI and waist circumference and the quality requirements of the trust meant that these aspects remained in the proforma.

Processes for transporting blood samples to the main hospital laboratory were clarified at peer meetings. It was made clear to doctors that a duty officer based at reception was available to transport bloods at all times. They could also be contacted via the bleep system. This meant that doctors could complete baseline bloods at any time but would have to ensure the samples are left with the duty officer for transport. Estates and facilities were contacted to service malfunctioning ECG machines and some were replaced.

The further refined proforma (see online supplementary Appendix 1c) was again piloted on the same two acute wards for a 4 -week period with the later 2 weeks being following changeover of doctors. Data from the second cycle demonstrated a further improvement in full physical health screens to the point where compliance was $90 \%$. Subsequent discussion at the MDT meeting concluded that $90 \%$ was an acceptable level of compliance and any additional cycles would likely yield limited further improvement due to other postulated reasons for lack of compliance, such as general attitudes to physical healthcare in mental healthcare, which could not be addressed within the remit of this project. It was also agreed that ECG, which was completed in $85 \%$ of cases at cycle 2 , should be a mandatory part of the admission process. The reduction in completion of the general screen by $12.5 \%$, which is more crucial if the full assessment could not be completed, was likely reflected by the fact that completion of the full physical health assessment increased.

During cycles, we gave presentations at all the local trust academic meetings to present the results and obtain continuous feedback from colleagues. At the time of cycle 2 , we were also involved in the induction of junior doctors informing them of the recent changes to the clerking proforma and educating them on the importance of managing physical health in mental health patients.

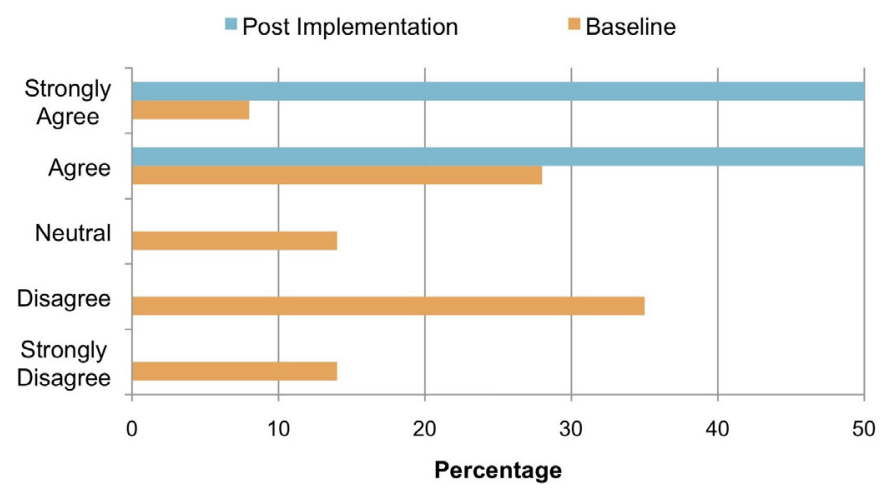

Figure 1 Results from doctor feedback survey question-'l find the admission process straightforward to complete'. 


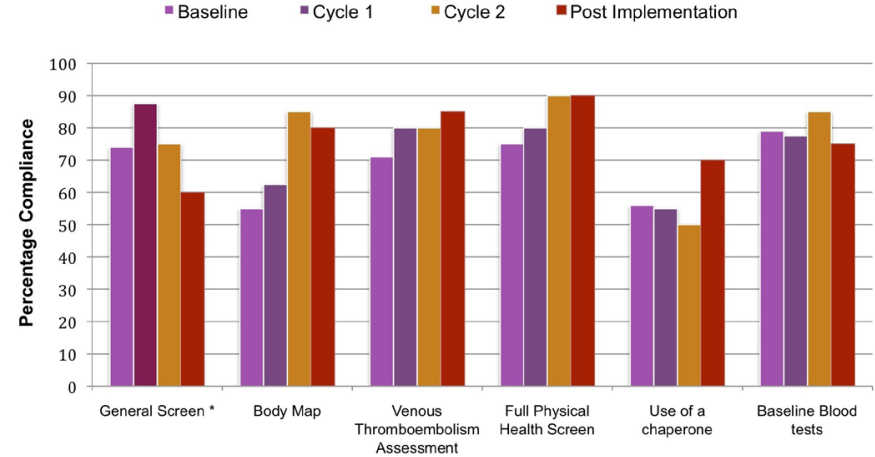

Figure 2 Bar chart showing percentage compliance with parameters of the physical admission process at baseline, postcycle 1, 2 and at postimplementation. *This includes a general physical observation of the patient, measurement of physical observations if available and documentation of their past medical history and allergy status.

Given the positive results obtained, the amended combined history taking and physical health proforma was approved by the safety and quality department to replace the prior doctors clerking documentation. This change was implemented trust wide.

\section{Post implementation data collection}

Compliance with the physical health checks was reaudited at 1 month postimplementation of the new and approved proforma. Data was collected using an excel spreadsheet and it demonstrated a sustained improvement in the full physical health check at $90 \%$ compliance. A further online survey was sent out to junior doctors, who were also in post during use of the prior clerking procedure and therefore in a position to give comparative feedback. Of those that responded ( $n=13), 100 \%$ found the process straightforward to use compared with $36 \%$ at baseline (figure 1), $90 \%$ found it easier to use than the previous proforma and all continued to agree that physical health checks are necessary on admission.

\section{RESULTS}

Key results for the baseline measurement, each PDSA cycle and reaudit are given in figure 2 and table 1 .

Over the two PDSA cycles compliance with completing the full physical health screen went up by $15 \%$ to a final rate of $90 \%$. Post implementation data collection at 1 month after the second PDSA cycle revealed that this improvement was sustained. Other areas of improvement that were sustained were use of a chaperone during the assessment and completion of the VTE assessment, for which overall compliances improved by $14 \%$ and $14.5 \%$, respectively.

Completion of the body map improved significantly from $55 \%$ to $85 \%$ during the PDSA cycles but decreased back down to $80 \%$ when measured post implementation of the final proforma though there remained an overall improvement. A decline in completion of the general health screen by $14 \%$ was seen. A marginal decrease in compliance of $4 \%$ was seen in completing baseline blood

Table 1 Completion of parameters of the physical admission process, as percentages and fraction of total number of cases for which each parameter was applicable, at baseline, PDSA 1, 2 and reaudit

\begin{tabular}{|c|c|c|c|c|}
\hline & Baseline $(n=139)$ & PDSA cycle $1(n=40)$ & $\begin{array}{l}\text { PDSA cycle } 2 \\
(n=20)\end{array}$ & $\begin{array}{l}\text { Reaudit } \\
(n=20)\end{array}$ \\
\hline General screen* & $74.1 \%(103 / 139)$ & $87.5 \%(35 / 40)$ & $75 \%(15 / 20)$ & $60 \%(12 / 20)$ \\
\hline $\begin{array}{l}\text { Venous thromboembolism } \\
\text { assessment }\end{array}$ & $70.5 \%(98 / 139)$ & $80 \%(32 / 40)$ & $80 \%(16 / 20)$ & $85 \%(17 / 20)$ \\
\hline Use of a chaperone & $56.1 \%(78 / 139)$ & $55 \%(22 / 40)$ & $50 \%(10 / 20)$ & $70 \%(14 / 20)$ \\
\hline Baseline blood tests§ & $79.1 \%(110 / 139)$ & $77.5 \%(31 / 40)$ & $85 \%(17 / 20)$ & $75 \%(15 / 20)$ \\
\hline Plan for reattempts if incomplete & $25.7 \%(9 / 35)$ & $45.5 \%(5 / 11)$ & $50 \%(2 / 4)$ & $50 \%(1 / 2)$ \\
\hline
\end{tabular}

${ }^{*}$ This includes a general physical observation of the patient (eg, mobility, alertness, overall wellness), measurement of physical observations if available and documentation of their past medical history and allergy status.

†Identifying body scars, bruises, piercings and tattoos.

‡Detailed physical examination of patient including cardiovascular, respiratory, abdominal and neurological examinations.

§Full blood count, urea and electrolytes, liver function tests, thyroid function, C reactive protein, B12, folate, bone profile, prolactin, cholesterol, HBA1c.

PDSA, plan do study act. 
tests. Completion of baseline ECG went up from $65 \%$ to $90 \%$.

It was noted that during cycle 1 after there was a changeover of doctors there was a significant drop in completion of the full physical health screen to $60 \%$, which was largely attributed to an individual doctor. To account for this bias, once the post changeover data were removed from the final analysis, there was an overall improvement in compliance to $80 \%$.

\section{LESSONS AND LIMITATIONS}

There are a number of valuable lessons learnt from this project. When reviewing previous interventions that have been implemented to improve physical health assessment in psychiatric patients, we found that none actually achieved a sustained result. Though educating junior doctors in the quality of good clerking is important, it did not result in the desired effect. Doing a doctor's questionnaire at the beginning of the project was extremely useful. The questionnaire revealed that all doctors felt a physical health clerking was necessary but found the existing proforma frustrating to use and therefore educating junior doctors again was not a suitable intervention.

Using the results of the questionnaire, we were able to come up with a new proforma and make continued improvements on it until both compliance and satisfaction with the proforma were optimal. Previous projects had not sought in detail the thoughts and opinions of those actually implementing the change but by including them as part of the project, we were able to achieve a change that junior doctors felt empowered by. We received a lot of positive feedback throughout the project as a lot of doctors felt this change was long overdue. Doctors found the new proforma to be more efficient, concise and easier to use, and it generated a lot of enthusiasm among our colleagues.

On achieving 90\% compliance, the MDT deemed this satisfactory, though we initially set out to achieve a $100 \%$ compliance. On discussion among the MDT and colleagues, it was clear that there continues to be a cultural aspect to this, whereby for various reasons, less emphasis is placed on physical healthcare in a mental healthcare setting despite it being an acute hospital. There was a limit to how much this could be addressed, as this is a complex task, which broadens beyond the scope of a trust-based project.

This project was quite inexpensive to conduct and implement. We did it in our own time outside of clinical commitments and the cost of printing new proformas and distributing it to the wards were minimal. It is likely the new proforma is actually cost effective as it is fewer pages and saves time for the on call doctor to complete though this was not quantitatively measured in the project.

One of the main limitations of this project is that the direct impact intervention had on the quality of patient care before and after was not directly measured. The impact on patient care was observed indirectly from better compliance with physical health checks, from which we hoped health complications were highlighted and subsequently acted on. We did not, however, look into whether better compliance to the proforma correlated in unearthing more health problems than prior to intervention or whether these were resolved by the doctors looking after the patients.

The reaudit conducted 1 month after the second PDSA cycle demonstrated a short-term sustained improvement in compliance with physical health assessments and we hope that the change in process itself will result in longterm improvement. However, we have not instituted measures to assess this, which would require at least an annual review. We strongly recommend that junior doctors are involved in this review and any process of change, which again should be done through an MDT for maximal effect. Further methods to improve spread and sustainability, some of which were used in this project, centre on dissemination of information to both clinical and non-clinical staff. This could include use of the trust newsletter, ongoing review in ward MDT meetings, which are a useful way to gain feedback, and involves long-term staff members such as ward managers and consultants as juniors rotate every 4-6 months. For new doctors to the trust, we would recommend incorporating a more detailed talk on the admission process and the standards that are expected into their induction week. To further reiterate this message, posters can be put up in the junior doctor's common areas and on the wards.

\section{CONCLUSION}

Physical healthcare of mental health patients at the point of admission within the trust was complicated by lack of a well-structured admission process. Focus on the physical health aspect as an independent part of the process alone did not produce significant results. However, implementation of a more holistic approach towards assessment based on traditional medical clerking procedures that all doctors are familiar with did significantly improve compliance with physical health checks. Importantly, doctors were pleased with the change likely contributing to a sustained improvement.

A new quality improvement approach provided a framework to test recommended changes and evolve design based on repeated data collection and MDT reviews between cycles. This also likely contributed to the sustained improvement in compliance seen as opposed to historical audit projects. The role of continuing to promote good physical healthcare and monitoring compliance has been handed over to successor colleagues.

Incorporation of physical healthcare into mental healthcare is key to achieving overall better quality of care in psychiatry, with the expectation that the gap in life expectancy in these patients compared with non-psychiatric patients will begin to reduce. 
Acknowledgements We would like to acknowledge Dr Faith Ndebele, Dr Bryn Williams, Dr Amy Blake and Susan Smith for their help in this project.

Contributors SH was the lead for the project, involved in all aspects including design, execution, data collection, analysis and implementation of recommendations. SB made significant contributions to the design of the plan-dostudy act cycles, data interpretation and implementation of recommendations. HA formulated the initial project idea and provided invaluable assistance in reviewing and editing the paper. All authors contributed to the writing of the paper.

Funding The authors have not declared a specific grant for this research from any funding agency in the public, commercial or not-for-profit sectors.

Competing interests None declared.

Patient consent for publication Not required.

Provenance and peer review Not commissioned; externally peer reviewed.

Open access This is an open access article distributed in accordance with the Creative Commons Attribution Non Commercial (CC BY-NC 4.0) license, which permits others to distribute, remix, adapt, build upon this work non-commercially, and license their derivative works on different terms, provided the original work is properly cited, appropriate credit is given, any changes made indicated, and the use is non-commercial. See: http://creativecommons.org/licenses/by-nc/4.0/.

\section{REFERENCES}

1. Chang C-K, Hayes RD, Perera G, et al. Life expectancy at birth for people with serious mental illness and other major disorders from a secondary mental health care case register in London. PLoS One 2011;6:19590.

2. DE Hert M, Correll CU, Bobes J, et al. Physical illness in patients with severe mental disorders. I. Prevalence, impact of medications and disparities in health care. World Psychiatry 2011;10:52-77.
3. Roberts L, Roalfe A, Wilson S, et al. Physical health care of patients with schizophrenia in primary care: a comparative study. Fam Pract 2007;24:34-40.

4. Brown S, Birtwistle J, Roe L, et al. The unhealthy lifestyle of people with schizophrenia. Psychol Med 1999;29:697-701.

5. Skala JA, Freedland KE, Carney RM, et al. Coronary heart disease and depression: a review of recent mechanistic research. Can $J$ Psychiatry 2006;51:738-45

6. De Hert M, Dekker JM, Wood D, et al. Cardiovascular disease and diabetes in people with severe mental illness position statement from the European psychiatric association (EPA), supported by the European association for the study of diabetes (EASD) and the European Society of cardiology (ESC). Eur Psychiatry 2009;24:412-24.

7. Tschoner A, Engl J, Laimer M, et al. Metabolic side effects of antipsychotic medication. Int J Clin Pract 2007;61:1356-70.

8. De Hert MA, van Winkel R, Van Eyck D, et al. Prevalence of the metabolic syndrome in patients with schizophrenia treated with antipsychotic medication. Schizophr Res 2006;83:87-93.

9. Ruschena D, Mullen PE, Burgess P, et al. Sudden death in psychiatric patients. Br J Psychiatry 1998;172:331-6.

10. Cournos F, McKinnon K, Sullivan G. Schizophrenia and comorbid human immunodeficiency virus or hepatitis $\mathrm{C}$ virus. J Clin Psychiatry 2005;66:27-33.

11. Mackin P, Bishop DR, Watkinson HMO. A prospective study of monitoring practices for metabolic disease in antipsychotic-treated community psychiatric patients. BMC Psychiatry 2007;7:28.

12. Phelan M, Stradins L, Morrison S. Physical health of people with severe mental illness. BMJ 2001;322:443-4.

13. NICE Guidance. Improving the physical health of people with serious mental illness: a quality improvement approach, 2017. Available: https://www.nice.org.uk/sharedlearning/improving-the-physicalhealth-of-people-with-serious-mental-illness-a-quality-improvementapproach [Accessed 27 Jan 2019]. 